\title{
A IMPORTÂNCIA DAS CARACTERISTICAS PESSOAIS DOS ESTUDANTES DE MEDICINA NA SUA EDUCAÇÃO
}

Luiz. Enrıcsto de Almeida Troncon*

\begin{abstract}
Resumo
Neste artigo são apresentados relatos resumidos dos principais trabalhos sobre "Característicaspcssoais de cstudantes de Medicina", divulgados cm scssĩo cspecífica de recente congresso intemacional de Educação Mćdica. Ncssa sessão, foram abordados aspectos como gêncro, filosofia de vida, "tradicionalismo", habilidades analíticas e de comunicação verbal c cscrita, rastreamento de estudantes com qualidades pessoais negativas c estilos de aprendizagem, entre outros. O autor comenta a importância de se considerar aspectos nào cognitivos cm várias elapas da Educação Mćdica. Salienta que a aplicação efetiva destc conccito vai dcpender do aumento do conhecimento nesta área. Assim sendo, investigaçōes sobre novos aspectos de atributos bem conhecidos dos estudantes, o descobrimento de novas características c cstudos sobre a utilidade dos instrumentos disponivis para a investigação deste assunto, são necessários. O cntendimento do papel das caractcrísticas pessoais dos cstudantes e a sua incorporação pclas cscolas mćdicas resultará em educação mais apropriada e efetiva.
\end{abstract}

\section{Palavras-chave:}

- Caracteristicas pessoais dos cstudantcs;

- qualidades pessoais:

- atributos não cognitivos:

- Educação Médica; admissão de cstudantes:

- avaliação.

\section{Introduça}

A qualidade da formação profíssional do mćdico ć detcrminada pela complexa interação entre fatores diversos, ligados à instituição formadora, ao como de cducadorcs c, cm cspecial, ao estudante. Dentre os fatores ligados ao estudantc. as suas características pessoais constituem, obviamente. um dos mais importantes, uma vez que estão intimamentc rclacionados com o aprendizado e o desempenlıo. Não obstante, as características pessoais dos estudantes de Medicina constituem um assunto pouco estudado e pouco valorizado no processo de Educaçĩo Médica.

Durante o recente crento, de alcance mundial, na área de Educaçĩo Mćdica, a Sïrth Ottawa Conference on Medical Education, realizado cm meados de 1994 , em Toronto, Ontário, Canadá, tive a oportunidade de participar como moderador e debatedor de sessão sobrc "Características dos Estudantcs". Tendo em vista a

\footnotetext{
* Professor Associado do Departiunénto de Clinica Médica; Vice-Presidente da Comissaño de (iraduaçuo, Faculdaule de Medicina de Ribcirão P'reto, Universidade de São Paulo)
}

relativa falta de destaque que este tópico tem recebido, julguci de interesse divulgar um relato sumariado das principais comunicaçōcs constantes daqucla scssão. bem como publicar neste artigo os comentários que apresentci ao final da discussĩo dos traballos.

\section{Relato (Jos Trabalhos}

\section{Filosofia de vida de estudantes de Medicina}

A filosofia de vida de uma pessoa pode ser conceituada como a base das suas atitudes e de suas açòes. A Dra. Ulla Joscphson. do Karolinska Institute. de Estocolmo, Suécia. investigou a fillosofia devida de 385 cstudantes de quatro diferentes estágios do curso médi$\mathrm{CO}^{5}$. Os resultados foram comparados com os obtidos em dois grupos distintos de profissionais médicos: investigadores clínicos e práticos gerais. Foi empregado um questionário especialmentc cstruturado para cstc fim. As respostas às difcrentcs questòcs, proccssadas por análise fatorial, permitiram a definição de três perfis de filosofia de vida: " cristã ou cspiritual". " cicntíficoreducionista" e "erolucionista". Os resultados indicaram que entre os estudantes de Medicina predominam os perlis de filosolia "cristi-tradicional" c. cm menor cscala, "cvolucionista". Ao contrário, os investigadores clínicos aprescntaram predominancia de filosofia "científico-reducionista". O gnupo de médicos práticos gerais apresentou resultados comparáv cis aos dos cstudantes, porćm com traço "evolucionista" menos predominantc. As implicaçòcs destes achados no aprendi zado c na escolla de carreiras foram discutidas.

Valor preditivo de dados pré-aldmissionais acerea de habilidades analíticas e de comunicação verbal e escrita

A escola médica da Universidade de Kentucky, nos Estados Unidos, cfetuou a partir de 1991, modificaçōes no teste admissional ( $\mathrm{K} / \mathrm{C} . \mathrm{T}$ - Hedical College Admisision T'est) de modo a passar a cstimular o deschvolvimento de habilidades analíticas e de comunicação $v$ crbal c cscrita. A Dra. Patricia Mayo c colaboradores ${ }^{*}$ investigaram o valor preditivo dos resultados obtidos por 82 cstudantes nestc testc admissional sobre o descmpcnho dos mesmos cm uma nova disciplina curricular, delincada para cnfatizar o uso de habilidades analíticas no mancjo de aspectos psicossociais. O desempenlıo na nova disciplina foi analisado por 14 preceptores que consideraram forma c contcudo $\mathrm{cm}$ ensaios escritos e comportamento cm sessōcs de traballıo $\mathrm{cm}$ gnupo. Análises de corrclação entre os dados pré-admissionais e os de desempenho na nova disciplina mostraram que as medidas pré-admissionais são preditivas do descmpenlo futuro. 


\section{Rastreamento de estudantes com características pessoais negativa}

As cscalas cmpregadas para avaliaçào de competências clinicas dos cstudantes de Medicina não enfatizam a caractcrização de qualidades pessoais positivas ou negativas. Na cscola mćdica Beer-Sheva, da Universidade $\mathrm{Bcn}-\mathrm{Gurion}, \mathrm{cm}$ Israel, foi introduzido, recentemente, um sistema de rastreamento de csludantcs com características pessoais negativas. Estc sistcma consta de: 1) incorporação de "questòcs-indicc" adcquadas às cscolas empregadas para avaliação global de compctências clinicas, pelos preceptorcs dos principais cstágios clínicos, c 2) designaçĩo de um profissional com a tarefa de avaliar, a longo prazo, os resultados de cada estudante e de acompanhar as medidas corrctivas adotadas nos casos de "cstudantes problcmáticos" identificados. Um c.icmplo de "questão-indice" seria: "Você ofercceria a cstc cstudante um cmprego em sua equipc"?".

No relato do Dr. Ziv IVcizman c scus colaboradores 12 , no periodo considcrado, foram identificados 12 "casosproblcmáticos" dentrc 146 estudantes acompanhados. Para cada um destcs "casos" foi designado um tutor. Da ação destcs rcsultou que somente 3 dos 12 "estudantcs problcmáticos" tivcram que ser encaminhados ì dircção da cscola para as providências cabiveis. Os autores concluiram que as escalas globais de avaliação de competências são inadequadas para identificar estudantes com qualidades pessoais ncgativas. A introdução de questòes-indice melhora csta avaliação $\mathrm{c}$ a designação de um profissional com a incumbência de dirigir o rastrcamento destes casos traz resultados benéficos.

\section{Estudantes "tradlicionais" versus "não tradlicionais}

Muitas das escolas médicas norte-americanas acolhem um contingente numcroso de cstudantes "nàotradicionais", identificados por várias caractcrísticas, como, por cxemplo, serem mais vellos, casados, já terem curso superior prévio c excrccrcm, ou já terem c.xcrcido, outras atividades profissionais. Nestc cstudo efeluado em Philadelphia (Medical College of Pennivy vania), a Dra. Linda Nieman c scus colaboradores 10 compararam o desempenlıo de estudantes "não-tradicionais" com cstudantes "tradicionais" em um exame clínico objetivo estruturado por estações, destinado a avaliar competência médica global c, cspccificamente, quatro categorias de competências: história clínica, crame físico, comunicaçĩo com pacientes c conhecimento mćdico. O grupo de 78 cstudantes "não tradicionais" cra mais velho e continha mais mulheres que o conjunto de 78 cstudantes " tradicionais" analisados. Os resultados das múltiplas comparaçòcs cfetuadas entre os dois gnupos rciclaram que os estudantes "não tradicionais", tendem a ter mellhor desempenho na obınção da história clinica, mas não há diferenças significativas cntre os dois grupos na competência médica gcral ou nas outras categorias estudadas.

\section{Gênero e habilidade de realizar entrevista médica}

Neste trabalho, o Dr. Andrew Zweifler e seus colaboradorcs $^{13}$, da Univcrsidade de Micliegan. em Ann Arbor, testaram a hipótese de que estudantes do sexo feminino têm desempenho superior a de seus colegas de classe do scro masculino. cm uma entrevista visando avaliar o risco de AIDS cm pacientes simulados. O grupo investigado constituiu-sc de 8.3 mullieres c de 130 homens que foram requisitados a efctuar a cntrciista cm i casos de pacicntcs simulados: um homem bisscxual, uma mulher hetcrosscrual c um homem hetcrosscxual. Cada um dos casos aprescntava um ou mais fatores comportamentais de risco para AIDS em sua história clinica. O descmpenho de cada estudante ao efetuar a entrevista foi avaliado empregando protocolos de obsernações contendo componentes para pontos cspecíficos da entrevista. Os cscorcs obtidos pelos cstudantes do scro feminino para o descmpcnho geral foram maiores do que os dos estudantes do scxo masculino. Alćm disso, as mulheres tivcram descmpcnho superior cm vários dos pontos especificos. como cmpatia. ausência de projeçào de valores, habilidades nào-verbais c uso de afîrmações transacionais. Nào só as mullıcres tiveram desempenho geral supcrior aos dos homens. nos 3 casos simulados, como tambćm a difcrença cntre os gêncros foi maior no caso simulado da mulher hcterossexual. Deste modo. foi possivel concluir quc estudantes de Medicina do sexo feminino têm descmpenho superior ao de seus colegas do sexo masculino em uma entrevista para avaliação de riscos para AIDS, particularmente quando o "caso" entrevistado é de uma mulher.

\section{Política de ação afĩmativa para apoio ao desempenho acalêmico de estudantes negros da África do Sul}

A political de segregaçào racial ("apartheid"). vigente oficialmente na Árica do Sul atć 1986. tere. no campo educiacional. duas caractcristicas marcantes: a) a proibição do ingresso de negros nas escolas médicas. c b) a imposição aos ncgros de um padrào de instriçĩo delibcradamentc inferior. de modo a incapaciti-los a progrcdir cm seus estudos. Segundo Rod Colbon' ', a escola médica da Universidade da Cidade do Cabo iniciou em 1988 uma política de ação afirmativa que garantia accsso à cducação mćdica de matriculantes negros e, também, permitia que os mesmos prossegıissem no curso regular. mesmo tendo resultados insatisfatórios. No entanto. cstas medidas foram inefícazes uma vez que um número muito redurido de estudantes negros foi admitido e scus resultados foram muito insitisfatórios. A partir de 1991. à csta politica de açìo afirmativa acoplaram-sc outras medidas, como: a) criaçào de um programa de apoio pedagógico, incluindo aprimoramcnto no uso da lingua inglesa: insiituição da figura de docentes monitores; c) instilucionalização de flexibilidade curricular $\mathrm{c}$ c) açòcs de suporte social aos estudantes negros. Estas providências resultaram $\mathrm{cm}$ aumento substancial do número de matriculas anuais de csludantcs negros que, em 1994, atingiu cerca de $3(0 \%$ dos ingressantes. Mais ainda, houve scnsivel progresso no desempenho dos estudantes negros. com mais de $68 \%$ deles atingindo as metas acadêmicas previstas. A 
comparação entre as duas fases da politica de ação afirmativa desenvolvida na Faculdade de Medicina da Universidade da Cidade do Cabo cnfatiza a necessidade de se incluir flexibilidade curricular, apoio acadêmico c suporte social para que objetivos propostos sejam alcançados.

\section{Estilos de aprendizalgem de estudantes de Medicina}

Vários modelos são usados para descrever os estilos de aprendizagem de estudantes universitários. Um modclo amplamente accito define trĉs perfis: "superficial", "profundo" e "realizador". A prevalĉncia dos difcrentes estilos ć determinada por caracteristicas do próprio estudante, pelo sistema de ensino c por caractcristicas departamentais. Nestc trabalho, oDr. R. I. Hilliard ${ }^{4}$, investigou a ocorrência destes difercntcs cstilos nos estudantes da Faculdadade de Medicina da Universidade de Toronto cmpregando, para tanto, instrumento validado. O perfil mais prevalente foi o do estilo "profundo", seguido do "superficial", que foi seguido do cstilo "realizador". Não houve diferenças entre classcs, sc.io ou estado civil. A idade c a cscolaridade prévia constituiram fatores importantes, uma vez que estudantcs mais velhos e aqueles com curso superior prćvio tinham traços mais fracos dos estilos "superficial" e "realizador". Detectaram-sc, ainda, importantes relaçòes cntrc métodos de estudo e estilos do aprendizagem. Estudantes mais interessados $\mathrm{cm}$ "aprender" e com maior motivação e organização para cstudar eram mais freqücntemente detcntores do estilo "profundo". Por outro lado, cstudantes mais intercssados em obter notas altas e que dispendiam maior número de horas estudando, apresentavam o cstilo "realizador". O medo de "fallhas" esteve freqüicntcmente associado ao estilo "supcrficial" de aprendizagem. Houve, também, alguma correlaçĩo entre cstilos dc aprendizagem e notas finais obtidas. Notas mais altas foram correlacionadas com o estilo "rcalizador". No cntanto, não houve correlação cntre notas obtidas c a ocorrência dos cstilos "superficial" ou "profundo". Deste modo, a caracterização de cstilos de aprendizado c o uso de instrumcntos especificos para a sua identificação foram útcis para o entendimento do modo pclo qual os estudantes de Medicina aprendem.

\section{Comentários}

A formação profissional de um bom mćdico é freqüentementc vista pela cscola médica, simplesmente, como uma questão de sclecionar os "mclhores" dentrc os candidatos à matricula no primeiro ano $\mathrm{c}$ propiciar a eles o cnsino e o treinamento adequados. De fato, os estudantes de Medicina em qualquer lugar do mundo são, realmcnte, pessoas de desempenlıo cscolar supcrior e, provavclmente, detêm as notas escolarcs mais altas, em comparaçào com as dos alunos de outros cursos de graduação da Universidade, principalmente quando se consideram os rcsultados admissionais ou pré-admissionais. Mesmo naquelas escolas médicas ondc se estabeleceram políticas especificas destinadas a dar oportunidades a pessoas oriundas de minorias étnicas ou religiosas, ou de grupos sociais, como vem cresccntemente ocorrendo cm outros paises "l os cstudantes selecionados são, cm geral, os de melhor desempcnho acadêmico

A questão da "qualidade" do ingressante, entrctanto, é habitualmente entendida cxclusivamente sob o prisma dos aspectos cognitivos. Consideram-sc, cm muitas escolas, as notas obtidas nos diferentes niveis prć-universitários c valoriza-se, especialmentc, o desempenho cm cxames destinados a medir especificamente o conliccimento acumulado durante longos períodos de cstudo.

Igualmente, durante o curso de graduação, os procedimentos de avaliação do cstudante cursando qualquer uma das escolas médicas do mundo ocidental contém, ainda, ĉnfase acentuada nas habilidades pertinentes ao dominio cognitivo ${ }^{7}$.

No entanto, comcça gradualmente a ser reconhecida a importância do papcl de um número variado de caracteristicas pessoais nas difcrentes etapas da cducação do médico. O conjunto diversificado das caracteristicas pessoais dos cstudantes incluem fatores relativamente bem conhccidos como idade, gêtnero, estado civil, origem racial e cultural, religião, ctc. que, certamente, descmpcnham um papel relevante no aprendizado c no descmpenho dos estudantes de Medicina. Entretanto, o conhecimento sobrc a participação de cada um destes fatores, isoladamente ou em intcração dinâmica, está muito longe de ser completamente dominado.

Por outro lado, a investigação científica no campo educacional começa a rcvelar outras caracteristicas pessoais mais profundas, bem como a descrever qualidades mais complexas, cụja importanncia merecc scr determinada $a^{3}$, Incluem estc conjunto, entre outros elementos, as características psicossociais, e de personalidade os diferentes estilos de aprendizadgem c os traços de personalidade determinantes de qualidades pessoais marcadamente negativas, tanto para a prática da profissão médica, quanto para a vida civil.

Em muitas escolas mćdicas inovadoras do Hemisfćrio Norte, a avaliação das caracateristicas pessoais, não-cognitivas, já cstá definitivamente incorporada nos procedimentos de admissão ao curso de Medicina?. Entrevistas pessoais, observaçōes do comportamento do candidato $\mathrm{cm}$ sessões de trabalho $\mathrm{cm}$ gnipo, análise detallhada dos "curricula vitac", o exame de tópicos específicos $\mathrm{cm}$ cartas autobiográficas escritas pelos candidatos constituem algums das inumeras técnicas destinadas a estimar com a maior acurácia possivel, quais os melhores candidatos, dominio de conliccimento e capacidade intelectual à partc. Reconhecc-se, nestas escolas, que o médico deve deter um conjunto variado de caractcrísticas, muitas das quais já devem cstar presentes no momento do ingresso ao curso médico.

Do mesmo modo, os moderios métodos de avaliação de compctências clinicas já incorporam a abordagem de caracteristicas pessoais increntes ao relacionamento empático e de técnicas válidas c fide- 
dignas para a detecção precoce de qualidades negativas, respecitoso com pacientes, familiares, colegas ou outros profissionais, a qualidade da compaixão. os atributos da (leterminação, da perseverança c da tolerância, entrc outros exemplos 2,6 . Tcm sido, também, crescentc a prcocupação de algumas cscolas médicas com o descnvolvimento como a desoncstidade, a falta de integridadc, os traços sociopáticos de personalidade etc.

Nesta sessão da Sixth Ollawa Conference on Meclical Eclucation foram apresentadas contribuições importantes ao conliccimento sobre o tema " Caractcristicas dos Estudautes". Aspectos como a prevalência de diferentcs filosofias de vida entre estudantcs de diferentes níveis ${ }^{5}$, a importância de se considerar habilidades analíticas c de comunicação verbal c cscrita na seleção de candidatos à escola médica ${ }^{\circledR}$, mćtodos para identificar cstudautes com qualidades pessoais negativas e medidas para abordar esta situaçiál', difercnças cntre estudantes "tradicionais"e "não tradicionais" quanto ao dcsempenho clínico' ${ }^{10}$, as relaçôes entre gêncro $\mathrm{c}$ a habilidade de realizar entrcvista médica específica ${ }^{13}$, as difcrentes cstratégias de uma cscola médica sul-africana para lidar com características desvantajosas criadas por uma politica nacional de "aparthcid"1 c a prevalência de diferentes estilos dc aprendizagem e a sua rclação com os hábitos de estudo e o descmpenho escolart.

Esses dados reflctem uma tendência geral em Educação, em valorizar c investigar os múltiplos determinantes do proccsso de aprendizagem e da formação $c$ do desenvolvimento do ser humano.,

No que diz respeito ao processo de formação profíssional, cssa tendência vem sendo expressa por mcio de estudos de fatores cada vez, menos centrados $\mathrm{cm}$ modelos de ensino e na atividade didática do professor c cada vez mais nas características do cstudante, enquanto sujeito que aprende.

Nesta perspectiva, redefinem-se, também, o perfil e o papel do profcssor, quc deisa de ser apenas um individuo que domina determinado conteúdo e algumas técnicas didáticas; deve tomar-se um educador que possui, além dos atributos descritos acima, uma compreensão mais ampla do processo de aprendizagem e das caractcristicas de seus alunos. Deve, tambćm, o professor exibir comportamentos que denotem o domínio de habilidades $\mathrm{c}$ atitudes, cuja incorporação pelos estudantes dependa muito da observação repetida da atuação profissional dc "modelos" adequados.

Entrctanto, parece-me claro que o dimensionamento adequado do papel das diferentes caracteristicas dos estudantes, na Educação Médica, vai depender do aumento do conliccimento disponivel sobre esse tópico. Neste sentido, ć oportuno delincar, pelo menos, três áreas de interesse ou linhas principais de investigação. A primeira delas inclui a descrição de novas calacterísticas c a investigação de novos aspectos de algumas das caractcristicas dos estudantes que já são razoavelmente bem conhecidas. A segunda árca rclaciona-se com a investigação sobrc a utilidade $\mathrm{c}$ o potencial dos instrumentos e técnicas cmpregadas para a cxploração das características dos estudantes. A terceira área que é, possivelmente. a mais importante, ainda que notarclmentc dificil, diz respcito à interaçìo entre o conhlecimento acumulado sobre cstc assunto e os diferentes componentes do processo cducacional em Mcdicina. Esta área está rclacionada com as sćrias implicaçòcs da diversidade das caracteristicas dos cstudantes em tópicos altamente relcrantes como: seleção c almissão de cstudantes, adaptação dos cstudantes ao curriculo, desenho de novos cursos, métodos educacionais $c$ abordagens curriculares, desenvolvimento de técnicas destinadas à modificação de caracteristicas dos cstudantes, aconselhamento sobre opęões de carreirai, treinamento vocacional e seleção para programas de formaçâo pós-graduada. entre outros.

As escolas mćdicas. $\mathrm{cm}$ todo o mundo. acham-sc constantementc sob a influência de fatorcs de ordem politica, social c financeira. que podem detenminar importantes modificaçòes em scus destinos institucionais c cm suas estratégias educacionais. A isto acresce-se 0 desalio representado pelas modificaçòes ambientais. pelo aparccimento de novas doenças ou pelo recrudescimento de condiçòcs mórbidas tidas como controladas. Este conjunto de fatores requer mudanças na Educaçĩo Médica e, freqücntementc. impòe a necessidade do desenvolvimento de novas habilidades e atitudes. Assim sendo, a resposta das escolas médicas a estes fatores somente será apropriada c cfetiva se houver o entendimento completo do papel das caracateristicas pessoais dos cstudantes de Mcdicina e se as instituiçõcs forcm capazes de produzir conhecimento novo sobre estes tópicos c de incorporá-los em suas atividades educacionais

\section{Agradecimentos:}

Agradeço à Profa. Dra. Maria de Lourdes Veronesi Rodrigues e à Pcdagoga Ana Raquel Lucato Cianflone pclas contribuiçòes of crecidas à claboraçào deste artigo.

\section{Summary}

Abridged abstracts of selected papers on "Characteristicis of Students" presented at a recently held international meeting on Seclical Eiducation are presented. A number of topics were covered, including gender, life philosophy, "traditionalism", analytic and communicalion skills, screening of medicalstudents with negative personal characteristics and learning styles: The importance of non-cognitive atributes in different stages of Hedical Education is stressied. Research on new aspects of well know characteristics, disclosure of new abilities and studies on methods for the investigation of personal characteristics of medical students are strongly needed. Production and incorporation of new knowledge on personal characteristics of stuclents will increase the appropriatedness and effectiveness of Medical Education.

\section{Key-Words:}

- Medical Suclents:

- Personal characteristics; 
- Non-cognitive variables;

- Medical Education;

- Admission of students assessment.

\section{Referências Bibliográficas}

1. COLBORN, R. The effect of affirmative action on medical student academic performance. The Sixth Ottawa Conference on Medical Educations, Toronto, Ontario, Canadá, 1994. Program Book, p. 27.

2. DAWSON-SAUNDERS, B.; PAIVA, R. A. - The validity of a clerkship performance evaluation. Medical education. 20: 24()-45, 1986.

3. HERMAN, M.W.; VELOSKI, J.I. - Premedical training, Personal characteristics and performance in Medical School. Medical education. 15 : 36367, 11981 .

4. HILLIARD, R. - Learning styles of undergraduate Medical Students. The Sixth Oltawa Confcrence on Mcdical Education, Toronto, Ontario, Canada, 1994. Program Book, p. 30.

5. JOSEPHSON, U. - Life-philosophy of medical students, Karolinska Institutc, Stockolm. The Sixth Ottawa Conference on Medical Education, Toronto, Ontario, Canada, 1994. Program Book, p. 27.

6. KECH, J.W.; AMOLD, L.; WILLOUGHBY, L; CALKINS, V. - Efficiency of cognitive/ noncognitive measures in predicting resident - phwsician performance. Journ. Mcd. Educ. 54: $1759-765,1979$.
7. LOWRY, S. - Assessment of Students. British Mcd.Jour. 306: 51-5, 1993.

8. MAYO, P.; ELAM, C.: JOHNSON, M.: REER, E. Use of preadmission variables to predict student performance in an interdisciplinary behavioral science course. The Sixth Ottawa Conference on Medical Education, Toronto, Ontario, Canada, 1992. Program Book, p. 27.

9. Mc GAGHIE. W. C. - Qualitative variables in Medical School admission. Ac.Med. 65:1459,- 199)().

10. NIEMAN, L. Z;; ROMANO, M.; GRACELY, E.: COHEN, D.; KELLIHER, G. J. -Comparison of traditional and non-traditional students clinical skills performance on an OSC.E. The Sixth Ottawa Conference on Mcdical Education, Toronto, Ontario, Canada, 1994. Program Book, p. 29.

11. PETERSDORF, R,G.: JURNER, K.; NICKENS, H.W.; READY, T. - Minoritics in Medicine: Past, Prcsent and Futurc. Acad. Mcdic. 65: 66367(), 199().

12. WEISMAN, Z.; BURGER, S.: MARGOLIS, C.Tracing of medical students with negative personal characteristics. The Sixth Ottawa Conference on Medical Education. Toronto. Ontario. Canada, 1994. Program Book, p.28.

13. ZWEIFFLER, A, J.: DICKEN, L. L.: WOLF, F. M. The importance of gender in the performance of an AIDS risk assessiment intervie'w. 'The Sixth Ottawa Conferenceon Medical Education, Toronto, Ontaro, Canada. 1994. Program Book, p.29.

Endereço do Autor

Departamento de Clinica Mćdica - Hosp. das Clinicas

Campus da USP

$14(0) 48-9(0)$ - Ribeirão Preto - SP

Tel: (016) 633-()43 Fax: (016) 633-11 44/ 633-6695 